\title{
Challenges in plasma edge fluid modeling
}

\section{R. Schneider, A. Runov}

Max-Planck-Institut für Plasmaphysik, EURATOM Assocoiation, 17491 Greifswald, Germany

E-mail: Ralf.Schneider@ipp.mpg.de

\begin{abstract}
Plasma fluid models like B2, UEDGE or EDGE2D are the standard tools for simulation of scrape-off layer physics, both for design and experimental support. The concept of a numerical tokamak, aiming at a predictive code for ITER, triggers the need to re-assess the available tools and their necessary extensions. These additional physics issues will be summarized. The experience existing in other scientific fields with multi-scale problems and modeling should be used as a guide. Here, the coupling strategies are in particular of interest for fusion problems. As a consequence, a certain construction of integrated modeling codes is needed: depending on the specific problem, models allowing different levels of complexity will be needed. Therefore, a hierarchy of tools is necessary, which will be discussed.
\end{abstract}




\section{Introduction}

Fluid models like B2, UEDGE or EDGE2D are the standard tools for simulation of scrape-off layer physics, both for design and experimental support [1-5].

The description of plasma-wall interactions, including the presence of walls and atomic physics processes within the plasma, is absolutely necessary for an understanding of edge plasmas $[6,7]$. The necessary building blocks for a model of the edge plasma are shown in Fig. 1.

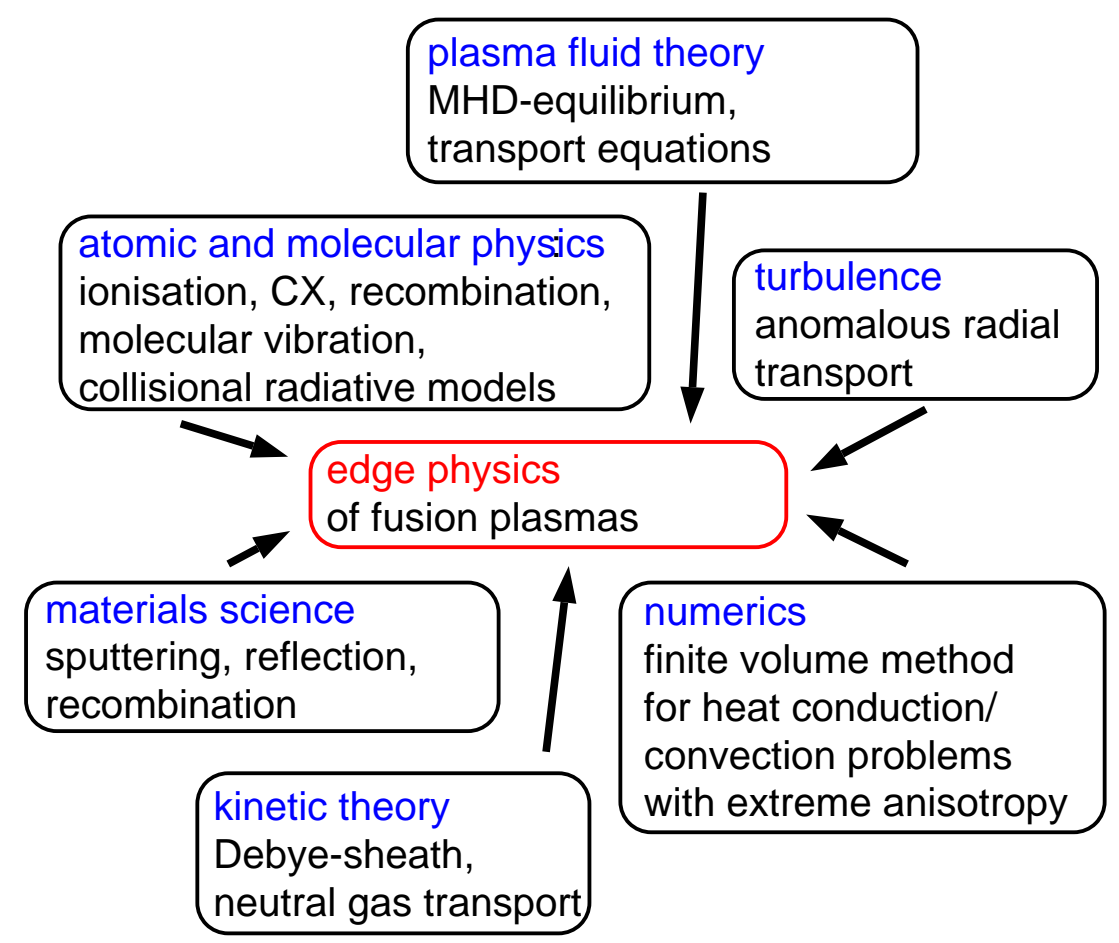

Figure 1. Components of a comprehensive edge plasma model.

The knowledge of the experimental magneto-hydrodynamic equilibrium is a necessary pre-requisite for any work in edge plasma physics. General plasma fluid theory is needed to derive the transport equations in the plasma edge region. Kinetic theory is necessary to obtain a correct description of parallel transport and the sheath that builds up in front of walls with which the plasma is in contact. This further defines boundary conditions to be used in the fluid model of the transport code. The interactions between plasma and walls also require a detailed understanding of the processes between the material surface and the plasma ions and neutrals (sputtering, reflection, recombination). Atomic and molecular processes have a major influence on the plasma through ionisation and recombination, for which collisional-radiative models are used (which even include vibrationally resolved molecules). The classical transport of neutrals strongly determines the plasma operating regime. In order to be able to optimize existing designs, the complete kinetic transport equation must be solved in full geometry. Because of the existence of turbulent radial transport in fusion experiments, 
one must include scaling laws or a direct coupling to turbulence codes in order to guarantee reliable predictions. Finally, one must also solve the numerical problems which arise from the extreme anisotropy of the transport equations (because of the very strong parallel electron heat conductivity), as well as combine all the above elements into a useful code, which should produce meaningful results on the basis of a very complex physics model within reasonable computer run times.

Plasma edge modeling made large progress over the last 20 years driven by the development of large code packages like B2-Eirene (now: SOLPS), UEDGE or EDGE2D [1-5]. These codes were developed to support existing machines for experiment interpretation and design optimization [8-16], but also to support the ITER design process [17-22].

Since about 1990 the list of physics to be included in such packages was agreed on [23-29] and step-by-step introduced:

- Parallel transport, including kinetic corrections;

- Perpendicular transport, based on turbulence theory or measurements;

- Inclusion of drifts and currents;

- Impurity physics: Generation (plasma-wall interaction) and transport (e.g. improvement of parallel transport model using 21-moments) of impurities;

- Neutral physics: Generation (plasma-wall interaction) and transport (full 3D Boltzmann equation including neutral-neutral collisions);

- Atomic and molecular physics: Reliable data for all processes (ionization, recombination, charge exchange, vibrational excitation of molecules, ... ), radiation transport;

- Inclusion of 3D effects: Limiters in the main chamber, effect of error fields and ergodic effects (close to X-point or in an ergodic layer).

The concept of a numerical tokamak, aiming at a predictive code for ITER, triggers the need to reassess the available tools and their necessary extensions. This paper will try to focus on the following basic questions:

What are the problems to be solved in edge modeling? Here, important open questions will be shortly discussed together with ideas and strategies to solve them.

Which strategies should be followed? Several possibilities of multi-scale coupling exist based on experience from other scientific fields, which will be summarized.

What are the consequences of these strategies? The idea of integrated modeling can be interpreted in very different ways, e.g. coupling of more and more sophisticated codes. This paper suggests and discusses a concept for integrated modeling with a flexible structure.

\section{Problems in edge modeling}

The goal to construct a predictive code for ITER and to develop a numerical tokamak needs to put further efforts into the improvement of existing tools. In the following, 
typical examples of actual problems in edge plasma modeling are presented.

\subsection{Plasma model}

The fluid equations are accepted and agreed upon for collisional regimes [30-36]. Special effort was necessary to complete the work for drifts and currents [37-49]. Here, a key element for a numerical implementation was the analytical removal of the divergence-free terms from the equations to guarantee a numerical robust scheme [44]. The equation for the electric potential is determined from the divergence-freeness of the currents (charge separation occurs on short kinetic scales, namely the Debye length, which is not included in fluid models). One remaining topic is here the discussion about the contributions in the radial currents $[42,46]$. Turbulence studies show that only anomalous viscosity - either Reynolds stress or corrections from gyro-viscosity - can create anomalous transport. Anomalous current resistivity cannot create anomalous transport or a radial electric field, because resistivity is a momentum-conserving friction between electrons and ions [50]. In the numerical performance, all codes still suffer from large reductions of time steps for regimes with large gradients when the drift terms can get very large. If the time step gets down to small values (typically $10^{-7} \mathrm{~s}$ ) the advantages of fully implicit schemes disappear and explicit methods might be a real alternative $[48,49]$. Further detailed analysis is needed, because otherwise only limited point studies are possible.

The kinetic effects can be important for the edge plasma modelling. There is an intrinsic kinetic problem here: to formulate boundary conditions on a material surface one needs some kind of kinetic treatment. Furthermore, due to operation at low densities for e.g. advanced scenarios, the problem of kinetic corrections and limits for parallel transport coefficients gets more and more important [3,51-53]. Usually, local limits of fluid transport coefficients are used. However, if these limits get important they can only indicate that full kinetic models are necessary. Another approach is to couple fluid codes with some simplified kinetic modelling: Andrei Kukushkin et al. [54] combined self-consistently 2D fluid transport model with two 1D kinetic equations in BGK-approximation to take account of kinetic effects for thermal conductivity, viscosity and boundary conditions. It should be noted that the perpendicular transport can also be affected: hot edge plasmas will be likely to exhibit potentially significant neoclassical ion transport and that plasma turbulence may also depend on kinetic effects beyond fluid theory. Here kinetic effects arise from radial coherence over scales larger than the gyroradius for (banana) transport and turbulence may have important variations on scales less than the gyroradius that also requires kinetic models [55,56]. An adjusting of both parallel and perpendicular transport coefficients of fluid models by coupling with kinetic codes might be one possible ansatz avoiding the need for kinetic codes resolving the full gyro-kinetic dynamics, which are extremely expensive in terms of computer time and memory.

The inclusion of 3D geometry effects is important for several processes in tokamaks: 
limiters in the main chamber are really 3D and studies of ramp-up scenarios for ITER have to take into account these effects [57]. Also, the 3D MHD effects in ergodic scenarios, like the use of additional perturbation coils in TEXTOR-DED or DIII-D, need a description. There are two levels of incorporation of ergodic effects into the edge models:

(i) using the 2D-codes mentioned before with some effective perpendicular diffusion coefficients like Rechester-Rosenbluth [58] or

(ii) direct 3D modelling of transport in ergodic configurations [59-61].

The first approach assumes an idealistic situation - full ergodicity without island remnants etc.; the penalties of the second approach are increased computational time and the need for local magnetic coordinates with a full metric tensor.

\subsection{Neutral model}

Depending on the question and the problem addressed, different complexity of this module is needed. One option is a full kinetic model solving the 3D Boltzmann equation with Monte Carlo $[2,62]$ with very different levels of physics implemented [63-65]: atoms experience ionization, charge-exchange and recombination. Molecules undergo dissociation and the effect of vibrational excitation is also included. For special cases non-linear effects of neutral-neutral collisions are needed. Alternatively, simplified fluid models [3,66-68] can supply a fast neutral model and still get reasonable accuracy. A typical example where the problem determines the tool needed is the study of the formation of the radial electric field in the pedestal region: a full kinetic neutral model takes too much run-time and details of the solution in the divertor are not really important for the pedestal physics in most of the cases, therefore fluid models are chosen. For realistic results, kinetic limits of the neutral transport coefficients (random walk determined by charge-exchange and ionization) need to be calibrated versus the full kinetic model. The classical problem of neutral transport is due to the existence of these different tools usually no problem and does introduce basic uncertainties in the edge model. However, a problem still remaining is the uncertainty in atomic and molecular cross-section data below about $5 \mathrm{eV}$. Also, the coupling of neutral Monte Carlo codes with finite volume plasma transport codes introduces noise in the coupled system, which requires additional diagnostics for convergence $[69,70]$.

\subsection{Turbulence}

This is the central unresolved topic and the remaining largest uncertainty in the plasma model. Again, a full spectrum of methods should be used

- Simplified fitting of experimental results for intelligent interpolation of measurements: Here, the 2D codes are used to fit the experimental profiles and diagnostics to allow an empirical determination of the anomalous transport coefficients [71]. 
This allows further analysis of scalings of the transport coefficients, e.g. density limit studies.

- Physics-based dependencies for playing physics (very important to see the consequences of certain theories or ideas for a more complete picture): Examples for this are the study of ballooning-like transport laws [72] or the implementation of increased radial transport as a characteristic of intermittent (blobby) transport in the far-outer scrape-off layer [73].

- Ab-initio turbulence models: Again, several levels should be tested from local parametrization of fluxes [74] to the full coupling of a transport and a turbulence code $[75]$.

\subsection{Plasma-wall interaction}

Another important topic is the inclusion of plasma-wall interaction processes. An example demonstrating the importance of these for the operation of a tokamak is the chemical erosion of carbon by hydrogen which produces hydrocarbon species [76]. Their dissociation and recombination in the plasma leads to amorphous hydrocarbon layer formation, which acts as sponge for hydrogen. Tritium is retained by co-deposition with carbon on the plasma facing sides or on remote areas, and this can lead to severe problems with the safety limit of about $1 \mathrm{~kg}$ of tritium in ITER.

Simple parametrization and fitting, e.g. by binary collision calculations of TRIM/TRIDYN for physical sputtering [77] or Roth's formula for chemical sputtering [78] is one possibility introducing these effects into edge models. More complex models like ERO [79] include the full kinetics near the wall. Finally, detailed studies for physics understanding of the chemical sputtering need complex molecular dynamics calculations [80].

\section{Multi-scale strategies and integrated modeling}

For multi-scale problems in general, either a serial coupling or a concurrent tight coupling as frequently as each time-step is used [81]. This depends on the problem one is interested in, which might require micro-scale information locally or globally. Examples for these different approaches are e.g. multi-scale modeling of hydrogen transport (parametrization of diffusion calculations from molecular dynamics for kinetic Monte Carlo is possible [82]) or calculations of plasma-enduced cracks in the divertor/wall material surfaces (using ab-initio methods only in the region close to the crack, otherwise using classical molecular dynamics [81]). The combination and coupling of more and more codes does not improve the reliability and predictive quality of such models, because the individual errors and uncertainties can amplify through the coupling.

Given the experience in multi-scale modeling in other disciplines, the following strategy for integrated modeling is suggested: One should have several physics modules with different levels of complexity for the individual problem blocks (standard, upgraded, 
downgraded). A general code combining all most complex sub-models is usually not needed, because this limits the time-steps and length-scales to the smallest ones in the problem. Instead, a very flexible tool should be created where the different modules of varying complexity can be combined depending on the question addressed and the physics of interest.

A good example is the continuing use of the 2-D codes as intelligent "interpolation" routines to complement experimental data for variables or regions where data does not exist (simple as possible for computational speed; e.g. no drifts and currents, simple neutral model) in contrast to full physics models, where a coupling with turbulence codes might be important (at least for proof-of-principle studies).

Another example, where a full integrated model is required to be able to get a realistic description is the problem of ELMs (Edge Localized Modes). These expel periodocally energy and particles from the hot core into the scrape-off layer and pose severe power load and erosion problems on the target plates. Here, MHD, turbulence, transport and plasma-wall interaction needes to be coupled for a full problem. Depending on the question addressed, different complexity levels are required for the edge model. Studies of core profile effects will not need a complete and detailed srcape-off layer model, whereas analysis of time-dependent power loads on the first wall and target plates and impurity erosion and co-deposition during ELMs will reuqire a full 3D transport code (even kinetic) and full hydrocarbon surface and bulk plasma chemistry. Details of a first step towards an integrated ELM model can be found in [83].

The flexibility of combination requires even more work for developing management and interface structures allowing such a flexible set-up. Not only technical support is needed but considerable effort by different specialists communicating with each other to clarify which levels need to be available for a particular question or problem.

Parallel to the module development several validation schemes have to be passed:

- Physics verification: The individual modules have to be checked against analytical test cases. For the coupling of different modules the physics and equations must be checked to avoid double-counting (e.g. inclusion of drift effects and calculation of radial electric field in the edge transport models, but also in turbulence codes, which one wants to couple with).

- Numerical verification: All codes must be freely accessible with full source code. Benchmarks between different codes and modules are needed. Codes with different numerical methods solving the same physics support the validation efforts. No code or module should be accepted which does not follow these standards.

- Experimental validation: Model systems are needed to benchmark and validate the code with experiments. The modular structures in the code should allow modular validation, which is very important, because with many different physics effects there is the danger that the uncertainties in all modules - either in terms of physics or numerics - will not allow a real significant result, because the uncertainties are adding up or even amplify too strongly. This defines the need for hierarchical 
benchmarking increasing the complexity of model systems and sensitivity studies for each problem addressed. Also, experimental model systems for these hierarchical checks are needed, e.g. linear devices for turbulence validation or low temperature plasmas and beam experiments for some aspects of the chemical sputtering problem.

\section{Summary}

In summary, one can state that complex problems like edge modeling and integrated modeling require complex tools with flexibility for different systems and questions in terms of complexity, numerics and physics. The paper presented a brief overview of challenges in edge plasma modeling, especially related to the integration of many physical processes. Such integrated systems should be flexible enough to produce both rapid, approximate assessments, as well as more detailed studies. The proposed effective strategy will guarantee such flexibility in a "hierarchical" integrated model of the edge plasma. Depending on the problem different levels of complexity of the ingredients should be available from simple analytical up to complete 3D numerical descriptions.

\section{References}

[1] B.J. Braams. A multifluid code for simulation of the edge plasma in tokamaks. Technical Report 68, Next European Torus, January 1987. NET Report No. 68, January 1987 (EURFU/XII-80/87/68).

[2] D. Reiter. The EIRENE code, version: Jan. 92 Users Manual. Technical Report Jül-2599, KFA Jülich, Germany, 1992.

[3] R. Schneider, X. Bonnin, K. Borrass, D.P. Coster, H. Kastelewicz, D. Reiter, V.A. Rozhansky, and B.J. Braams. Plasma edge physics with b2-eirene. Contrib. Plasma Phys., 46:3-191, 2006.

[4] T.D. Rognlien, J.L. Milovich, M.E. Rensink, and G.D. Porter. A fully implicit, time dependent 2-D fluid code for modeling tokamak edge plasmas. J. Nucl. Mater., 196-198:347-351, 1992.

[5] R. Simonini, G. Corrigan, G. Radford, J. Spence, and A. Taroni. Models and numerics in the multi-fluid 2-D edge plasma code EDGE2D/U. Contrib. Plasma Phys., 34(2-3):368-373, 1994. 4th Workshop on Plasma Edge Theory, October 1993, Varenna, Italy.

[6] P.C. Stangeby. The Plasma Boundary of Magnetic Fusion Devices. IOP, Bristol, 2000.

[7] D.E. Post and R. Behrisch, editors. Physics of Plasma-Wall Interactions in Controlled Fusion, volume 131 of NATO Advanced Science Institute, New York, 1986. NATO Science Committee, Plenum Press.

[8] L.D. Horton and JET Team. Modelling and measurements of jet divertor plasmas. In 15th IAEA Conference on Plasma Physics and Controlled Nuclear Fusion Research, Seville, Spain, 26.September-1.October 1994, volume 1, pages 541-551, Vienna, 1995. IAEA. paper IAEA-CN60/A4-5.

[9] A. Loarte, A.S. Kukushkin, H.D. Pacher, D.P. Coster, R. Schneider, N. Asakura, K. Itami, B. LaBombard, B. Lipschultz, C.F. Maggi, R.D. Monk, G.D. Porter, M. Shimada, M. Sugihara, and J. Terry. Comparison of B2-EIRENE calculations with multi-machine experimental measurements. J. Nucl. Mater., 269:1123-1128, 1999.

[10] R. Schneider, H.-S. Bosch, D.P. Coster, J.C. Fuchs, J. Gafert, G. Haas, A. Herrmann, M. Kaufmann, A. Kallenbach, J. Neuhauser, J. Schweinzer, U. Wenzel, and ASDEX Upgrade Team. Role of divertor geometry on detachment in ASDEX Upgrade. J. Nucl. Mater., 266269:175-181, 1999. 13th PSI, San Diego, CA, USA, 18-22 May 1998. 
[11] G.C. Vlases, L.D. Horton, G.F. Matthews, P. Andrew, K. Borrass, A. Chankin, S. Clement, G. Conway, S. Davies, J. Ehrenberg, G. Fishpool, H.Y. Guo, P.J. Harbour, L.C. Ingesson, H.J. Jackel, J. Lingertat, A. Loarte, C.G. Lowry, C.F. Maggi, G.M. McCracken, R. Mohanti, R.D. Monk, R. Reichle, E. Righi, R. Smith, M.F. Stamp, P.C. Stangeby, A. Taroni, and M. von Hellermann. The effect of divertor geometry on divertor and core plasma performance in JET. J. Nucl. Mater., 269:160-167, 1999. 13th PSI, San Diego, CA, USA, 18-22 May 1998.

[12] D.N. Hill, M.E. Rensink, A. Futch, D. Buchenauer, T.W. Petrie, M.A. Mahdavi, and G. Matthews. Measurement and modeling of the DIII-D divertor plasma. J. Nucl. Mater., 176-177:158-164, 1990. 9th PSI Conference, Bournemouth, UK, 21.-25. May 1990.

[13] M.E. Fenstermacher, G.D. Porter, M.E. Rensink, T.D. Rognlien, S.L. Allen, D.N. Hill, C.J. Lasnier, T. Leonard, and T. Petrie. UEDGE and DEGAS modeling of the DIII-D scrape-off layer plasma. Journal of Nuclear Materials, 220-222:330-335, 1995.

[14] R.A. Pitts, B.P. Duval, A. Loarte, J.-M. Moret, J.A. Boedo, D.P. Coster, I. Furno, J. Horacek, A.S. Kukushkin, D. Reiter, J. Rommers, and The TCV Team. Divertor geometry effects on detachment in TCV. J. Nucl. Mater., 290-293:940-946, 2001. 14th PSI, Rosenheim, 22.-26. May 2000.

[15] G.D. Porter, T.D. Rognlien, M.E. Rensink, H. Takenaga, N. Asakura, A. Loarte, G. Matthews, and contributors to the EFDA-JET workprogramme. Simulation of the effect of plasma flows in DIII-D, JET, and JT-60U. J. Nucl. Mater., 313-316:1085-1088, 2003. 15th PSI, Gifu, Japan, 27.-31. May 2002.

[16] D.P. Stotler, R.A. Vesey, D.P. Coster, C.F.F. Karney, B. LaBombard, B. Lipschultz, C.S. Pitcher, and R. Schneider. Coupled Monte Carlo neutral fluid plasma simulation of Alcator C-Mod divertor plasma near detachment. J. Nucl. Mater., 269:947-952, 1999.

[17] A. Taroni, G. Corrigan, R. Simonini, J. Spence, and S. Weber. A study with the EDGE2D code of the power exhaust problem in ITER relevant divertor plasmas. J. Nucl. Mater., 220-222:10861090, 1995.

[18] R. Schneider, D. Reiter, D.P. Coster, J. Neuhauser, K. Lackner, and B.J. Braams. Analysis of cold divertor concepts for ITER. J. Nucl. Mater., 220-222:1076-1080, 1995. 11th PSI, Mito, Japan, 23-27 May 1994.

[19] A.S. Kukushkin, H.D. Pacher, D.P. Coster, G. Janeschitz, D. Reiter, and R. Schneider. 2D modelling of the edge plasma in ITER. Contrib. Plasma Phys., 38(1/2):20-25, 1998. 6th Workshop on Plasma Edge Theory, Exeter College, Oxford, UK, 15-17 September 1997.

[20] A.S. Kukushkin, H.D. Pacher, G. Janeschitz, A. Loarte, D.P. Coster, G. Matthews, D. Reiter, R. Schneider, and V. Zhogolev. Basic divertor operation in ITER-FEAT. Nucl. Fusion, 42(2):187-191, 2002.

[21] F. Wising, D.A. Knoll, S.I. Krasheninnikov, T.D. Rognlien, and D.J. Sigmar. Simulation of detachment in ITER-geometry using the UEDGE code and a fluid neutral model. Contrib. Plasma Phys., 36(2-3):309-313, 1996.

[22] ITER Physics Basis Editors and ITER Physics Expert Group Chairs and Co-Chairs and ITER Joint Central Team and Physics Integration Unit and ITER Physics Expert Group on Confinement and Transport and ITER Physics Expert Group on Confinement Modelling and Database and ITER Physics Expert Group on Disruptions, Plasma Control and MHD and ITER Physics Expert Group on Divertor and ITER Physics Expert Group on Divertor Modelling and Database and ITER Physics Expert Group on Energetic Particles, Heating and Current Drive and ITER Physics Expert Group on Diagnostics . Chapter 1-9. Nucl. Fusion, 39(12):2137-2638, 1999.

[23] M. Keilhacker, K. Lackner, K. Behringer, H. Murmann, and H. Niedermeyer. Plasma boundary layer in limiter and divertor tokamaks. Phys. Scripta, T2/2:443-453, 1982.

[24] D.E. Post, K. Borass, J.D. Callen, S.A. Cohen, J.G. Cordey, F. Engelmann, N. Fujisawa, M.F.A. Harrison, J.T. Hogan, H.J. Hopman, Y. Igitkhanov, O. Kardaun, S.M. Kaye, S.I. Krasheninnikov, A. Kukushkin, V. Mukhovativ, W. Nevins, A. Nocentini, G.W. Pacher, H.D. 
Pacher, V.V. Parail, L.D. Pearlstein, L.J. Perkins, S. Putvinskij, K. Riedel, D.J. Sigmar, M. Sugihara, D.W. Swain, T. Takizuka, K. Tani, T. Tsunematsu, N.A. Uckan, J.G. Wegrowe, J. Wesley, S. Yamamoto, R. Yoshino, K. Young, P.N. Yushmanov, and international contributors. ITER Physics. Number 21 in ITER Documentation Series. International Atomic Energy Agency, 1991.

[25] D. Reiter. Progress in two-dimensional plasma edge modelling. J. Nucl. Mater., 196-198:80-89, 1992.

[26] P.C. Stangeby, G.M. McCracken, and G.M. Staebler. Plasma boundary phenomena in tokamaks. Nucl. Fusion, 30:1225-1379, 1990.

[27] E.L. Vold. Multidimensional and multifluid plasma edge modelling status and new directions. Contrib. Plasma Phys., 32(3-4):404-421, 1992.

[28] K. Lackner and M. Keilhacker. Divertor physics: Summary of modelling and experiments. J. Nucl. Mater., 128-129:368-377, 1984.

[29] K. Lackner and R. Schneider. The role of edge physics and confinement issues in the fusion reactor. Fusion Eng. Des., 22:107-116, 1993. SOFT 92, Rom.

[30] S.I. Braginskii. Transport processes in a plasma. In Reviews of Plasma Physics, volume 1, pages 205-311. Consultants Bureau, New York, New York, 1965.

[31] A.B. Mikhailovskii and V.S. Tsypin. Transport equations of plasma in a curvilinear magnetic field. Beit. Plasmaphys., 24(4):335-354, 1984.

[32] A.A. Galeev and R.Z. Sagdeev. Transport phenomena in a collisionless plasma in a toroidal magnetic system. Sov. Phys. JETP-USSR, 26(1):233-240, 1968.

[33] A. Bergmann, Y. Igitkhanov, B.J. Braams, D.P. Coster, and R. Schneider. Implementation into B2 of a 21 moment description for the parallel impurity transport. Contrib. Plasma Phys., 36(23):192-196, 1996. 5th Workshop on Plasma Edge Theory, December 1995, Asilomar, California.

[34] G.J. Radford. The application of moment equations to scrape-off layer plasmas. Contrib. Plasma Phys., 32(3-4):297-302, 1992.

[35] Y.L. Igitkhanov. Impurity transport at arbitrary densities in the divertor plasma. Contrib. Plasma Phys., 28(4-5):477-482, 1988.

[36] V.M. Zhdanov. Transport processes in multicomponent plasma. Plasma Phys. Contr. F., 44(10), 2002. re-edition of a Russian document (first published in 1982).

[37] M. Baelmans, D. Reiter, R.R. Weynants, and R. Schneider. Computational assessment of effects of electric fields and currents in tokamak edge plasmas. J. Nucl. Mater., 220-222:982-986, 1995. 11th PSI, Mito, Japan, 23-27 May 1994.

[38] V.A. Rozhansky, S.P. Voskoboynikov, E.G. Kaveeva, D.P. Coster, and R. Schneider. Simulation of tokamak edge plasma including self-consistent electric fields. Nucl. Fusion, 41(4):387-401, 2001.

[39] A.V. Chankin and P.C. Stangeby. The effect of the diamagnetic drift on the boundary conditions in tokamak scrape-off layers and the distribution of plasma fluxes near the target. Plasma Phys. Contr. F., 36(9):1485-1499, 1994. Preprint in JET-P(94)18, 1994.

[40] A.V. Chankin, J.P. Coad, G. Corrigan, S.J. Davies, S.K. Erents, H.Y. Guo, G.F. Matthews, G.J. Radford, J. Spence, P.C. Stangeby, and A. Taroni. Modelling of SOL flows and target asymmetries in JET field reversal experiments with EDGE2D code. Contrib. Plasma Phys., 40(3-4):288-294, 2000. 7th PET, Tajimi, Gifu, Japan, 4.-6. October 1999.

[41] G.D. Porter, T.D. Rognlien, M.E. Rensink, H. Takenaga, N. Asakura, A. Loarte, G. Matthews, and contributors to the EFDA-JET workprogramme. Simulation of the effect of plasma flows in DIII-D, JET, and JT-60U. J. Nucl. Mater., 313-316:1085-1088, 2003. 15th PSI, Gifu, Japan, 27.-31. May 2002.

[42] V.A. Rozhansky, S.P. Voskoboynikov, E. Kovaltsova, D.P. Coster, and R. Schneider. Perpendicular conductivity and self-consistent electric fields in tokamak edge plasma. Contrib. Plasma Phys., 40(3-4):423-430, 2000.

[43] A.V. Chankin, G. Corrigan, S.K. Erents, G.F. Matthews, J. Spence, and P.C. Stangeby. 
Interpretation of SOL flows and target asymmetries in JET using EDGE2D code calculations. J. Nucl. Mater., 290-293:518-524, 2001.

[44] G.J. Radford, A.V. Chankin, G. Corrigan, R. Simonini, J. Spence, and A. Taroni. The particle and heat drift fluxes and their implementation into the EDGE2D transport code. Contrib. Plasma Phys., 36(2-3):187-191, 1996. 5th Workshop on Plasma Edge Theory, December 1995, Asilomar, USA.

[45] T.D. Rognlien, D.D. Ryutov, and N. Mattor. Calculation of 2-D profiles for the plasma and electric field near a tokamak separatrix. Czech. J. Phys., 48(S2), 1998. CD-ROM: Proceedings of the Edge-Plasma Theory and Simulation Workshop.

[46] T.D. Rognlien, D.D. Ryutov, N. Mattor, and G.D. Porter. Two-dimensional electric fields and drifts near the magnetic separatrix in divertor tokamaks. Phys. Plasmas, 6(5):1851-1857, 1999.

[47] V.A. Rozhansky, E.G. Kaveeva, S.P. Voskoboynikov, A.H. Bekheit, D.P. Coster, X. Bonnin, and R. Schneider. Impact of $\vec{E} \times \vec{B}$ drifts on the distribution of impurities in the tokamak plasma edge. J. Nucl. Mater., 313-316(3):1141-1149, 2003. 15th PSI, Gifu, Japan, 27.-31. May 2002.

[48] R. Zagorski, H. Gerhauser, and H.A. Claassen. Numerical simulation of the TEXTOR edge plasma including drifts and impurities. Contrib. Plasma Phys., 38(1-2):61-66, 1998.

[49] R. Zagórsky, H. Gerhauser, B. Bienkowska, and H.A. Claaßen. Modelling of Drifts and Currents in the Edge Plasma. Institut für Plasmaphysik Euratom Association, Trilateral Euregio Cluster, Berichte des Forschungszentrum Jülich GmbH, Jül-3829, 2001.

[50] B. Scott. The character of transport caused by ExB drift turbulence. Phys. Plasmas, 10:963-993, 2003.

[51] W. Fundamenski. Parallel heat flux limits in the tokamak scrape-off layer. Plasma Phys. Contr. F., 47:R163-R208, 2005.

[52] J.F. Luciani, P. Mora, and J. Virmont. Nonlocal heat transport due to steep temperature gradients. Phys. Rev. Lett., 51(18):1664-1667, 1989.

[53] J.P. Matte and J. Virmont. Electron heat flow transport down steep temperature gradients. Phys. Rev. Lett., 49(26):1936-1939, December 1982.

[54] Y.L. Igitkhanov, A.S. Kukushkin, A.M. Runov, and R. Chodura. Application of BGK collision operator for kinetic correction of fluid models. Contrib. Plasma Phys., 34(2-3):216-220, 1994. 4th PET, Varenna, Italy, October 1993.

[55] T. Rognlien. Progress on a continuum kinetic code for edge plasmas. This workshop.

[56] C.S. Chang. Us scidac program on integrated simulation of edge transport in fusion plasmas, and its progress. This workshop.

[57] M. Kobayashi. Power load analysis on the iter start-up limiter modules. submitted.

[58] K.H. Finken, T. Eich, S.S. Abdullaev, A. Klaeck, G. Mank, D. Reiser, A.M. Runov, and M. Tokar. Modeling of the dynamic ergodic divertor of TEXTOR-94. J. Nucl. Mater., 266-269:495-500, 1999. 13th PSI, San Diego, CA, USA, 18.-22. May 1998.

[59] N.A. McTaggart, X. Bonnin, A.M. Runov, R. Schneider, and R. Zagorski. Energy transport modelling including ergodic effects. Contrib. Plasma Phys., 44(1-3):31-34, 2004. 9th PET, San Diego, California, USA, 3-5 September 2003.

[60] A.M. Runov, S. Kasilov, D. Reiter, N.A. McTaggart, X. Bonnin, and R. Schneider. Transport in complex magnetic geometries: 3D modelling of ergodic edge plasmas in fusion experiments. $J$. Nucl. Mater., 313-316(3):1292-1297, 2003. 15th PSI, Gifu, Japan, 27-31 May 2002.

[61] A. Runov, S. Kasilov, R. Schneider, and D. Reiter. Extensions of the 3D plasma transport code E3D. Contrib. Plasma Phys., 44(1-3):18-24, 2004. 9th PET, San Diego, California, USA, 3-5 September 2003.

[62] D. Heifetz, D. Post, M. Petravic, J. Weisheit, and G. Bateman. A Monte-Carlo model of neutralparticle transport in diverted plasmas. J. Comput. Phys., 46:309-327, 1982.

[63] D. Reiter, P. Bachmann, and A.K. Prinja. Influence of elastic collisions on neutral gas transport in boundary plasmas. Contrib. Plasma Phys., 32(3-4):261-267, 1992. 3rd Workshop on Plasma Edge Theory, June 1992, Bad Honnef, FRG. 
[64] D. Reiter, C. May, D.P. Coster, and R. Schneider. Time dependent neutral gas transport in tokamak edge plasmas. J. Nucl. Mater., 220-222:987-992, 1995. 11th PSI Mito, Japan, 23-27 May 1994.

[65] D. Reiter. Neutral gas transport in fusion devices: Atomic and surface data aspects. In Atomic and Plasma - Material Interaction Processes in Controlled Thermonuclear Fusion, pages 243-266. Elsevier Science Publishers B.V., 1993.

[66] F. Wising, D.A. Knoll, S.I. Krasheninnikov, T.D. Rognlien, and D.J. Sigmar. Simulation of detachment in ITER-geometry using the UEDGE code and a fluid neutral model. Contrib. Plasma Phys., 36(2-3):309-313, 1996.

[67] M.V. Umansky, T.D. Rognlien, M.E. Fenstermacher, M. Borchardt, A. Mutzke, J. Riemann, R. Schneider, and L.W. Owen. Modeling of localized neutral particle sources in 3D edge plasmas. J. Nucl. Mater., 313-316(3):559-563, 2003. 15th PSI, Gifu, Japan, 27-31 May 2002.

[68] J. Riemann, M. Borchardt, R. Schneider, A. Mutzke, T.D. Rognlien, and M.V. Umansky. NavierStokes neutral and plasma fluid modelling in 3D. Contrib. Plasma Phys., 44(1-3):35-38, 2004. 9th PET, San Diego, California, USA, 3-5 September 2003.

[69] G.P. Maddison and D. Reiter. Recycling source terms for edge plasma fluid models and impact on convergence behaviour in the braams b2 code. Technical Report Jül-report 2872, Forschungszentrum Jülich, Germany, March 1994.

[70] M.E. Rensink, G.D. Porter, T.D. Rognlien, D.P. Coster, R. Schneider, C.F.F. Karney, and D.P. Stotler. Edge plasma simulations with coupled fluid and Monte Carlo models. Technical Report UCRL-JC-124364-ABS, Lawrence Livermore National Laboratory, Livermore, CA, 1996. Annual Meeting, American Physical Society (APS), 38th, Division of Plasma Physics, Denver, CO, November 11.-15., 1996.

[71] D.P. Coster, J.W. Kim, G. Haas, B. Kurzan, H. Murmann, J. Neuhauser, H. Salzmann, R. Schneider, W. Schneider, J. Schweinzer, and the ASDEX Upgrade Team. Automatic evaluation of edge transport coefficients with B2-SOLPS5.0. Contrib. Plasma Phys., 40(34):334-339, 2000. 7th PET, Tajimi, Gifu, Japan, 4.-6. October 1999.

[72] D.P. Coster, X. Bonnin, B. Braams, D. Reiter, R. Schneider, and the ASDEX Upgrade Team. Simulation of the edge plasma in tokamaks. Phys. Scripta, T108:7-13, 2004. Hydrogen in Condensed Matter 2003 Workshop, Helsinki.

[73] A.Yu. Pigarov, S.I. Krasheninnikov, T.D. Rognlien, W.P. West, B. LaBombard, B. Lipschultz, R. Maingi, and V. Soukhanovskii. Multi-fluid code simulations including anomalous nondiffusive transport of plasma and impurities in the tokamak sol. Contrib. Plasma Phys., 44(13):228-234, 2004.

[74] Y. Nishimura, D.P. Coster, J.W. Kim, and B.D. Scott. Coupling of perpendicular transport in turbulence and divertor codes. Contrib. Plasma Phys., 42(2-4):379-383, 2002. 8th PET, Espoo, Finland, 10.-12. September 2001.

[75] M.V. Umansky and T.D. Rognlien. Simulation of plasma fluxes to material surfaces with selfconsistent edge turbulence and transport for tokamaks. J. Nucl. Mater., 337-339:327-331, 2005.

[76] G. Federici, J.N. Brooks, D.P. Coster, G. Janeschitz, K. Krieger, A. Kukushkin, A. Loarte, H.D. Pacher, J. Roth, J. Stober, C.H. Wu, and H. Wuerz. Assessment of erosion and tritium codeposition in the reduced-size ITER. J. Nucl. Mater., 290-293:260-265, 2001. 14th PSI, Rosenheim, 22-26 May 2000.

[77] W. Eckstein. Computer simulation of ion-solid interactions. In Springer Series in Materials Science, Vol. 10. Springer, Berlin, 1991.

[78] J. Roth and C. García-Rosales. Analytic desciption of the chemical erosion of graphite by hydrogen ions. Nucl. Fusion, 36(12):1647-1659, 1996.

[79] A. Kirschner, J.N. Brooks, V. Philipps, P. Wienhold, A. Pospieszczyk, R.K. Janev, and U. Samm. Modelling of the transport of methane and higher hydrocarbons in fusion devices. J. Nucl. Mater., 313-316:444-449, 2003. 15th PSI, Gifu, Japan, 27.-31. May 2002.

[80] E. Salonen. Overview of the atomistic modeling of the chemical erosion of carbon. Phys. Scripta, 
Workshop on Edge Transport in Fusion Plasmas, 11-13 September, Krakow, Poland 13

T111:133-137, 2004.

[81] W. E Engquist and B. Engquist. The heterogeneous multi-scale methods. Comm. Math. Sci., 1:87-133, 2003.

[82] M. Warrier, R. Schneider, E. Salonen, and K. Nordlund. Multi-scale modeling of hydrogen isotope diffusion in graphite. Contrib. Plasma Phys., 44(1-3):307-310, 2004. 9th PET, San Diego, California, USA, 3-5 September 2003.

[83] A.H. Kritz and C.S. Chang. Modeling of elm dynamics in iter. This workshop. 